\title{
Japan pulls its fusion research together ...
}

Tokyo

Japan is to link ten nuclear-fusion research institutes into a single network to boost collaboration between university research and government-run institutes.

The move is being seen as a bid to increase Japan's chance of hosting the International Thermonuclear Experimental Reactor (ITER), the proposed next-generation fusion facility. Japan is widely considered to be the most likely candidate to host ITER, as it is expected to provide the largest share of the construction cost.

The new network is the product of the Science Council of the Ministry of Education, Science, Sports and Culture (Monbusho), and links previously independent efforts in fusion research at institutes run by Monbusho and the Science and Technology Agency (STA).

The move brings together senior researchers and directors of institutes such as Monbusho's National Institute for Fusion Science (NIFS) and STA's Japanese Atomic Energy Research Institute (JAERI), and will launch research plans in seven areas. These include the development of new material for reactor-vessel walls, the creation of a database for superconductor technology and the development of novel laser technology.

The collaboration also involves the Japan Nuclear Cycle Development Institute and the universities of Kyoto, Tokyo, Okayama, Tohoku, Tsukuba, Kyushu and Osaka. It is seen as a necessary step towards the impending merger of STA and Monbusho in 2001, and the planned transformation of national universities and research institutes into 'agencies' with greater administrative independence (see Nature 401, 416; 1999).

Osamu Motojima, professor of plasma research at NIFS, says that Japan's fusion programmes have been carried out as isolated efforts because of bureaucratic barriers between ministries. "The merger of STA and Monbusho should help break down the barriers and allow efficient collaboration between government institutes and universities, although the merger of institutes themselves (JAERI and NIFS) should be approached more carefully," says Motojima.

"A strong research base is necessary for Japan's future role in ITER, and it is therefore essential to build a stronger link between current domestic fusion programmes," says Masami Nakamura, director of STA's nuclear fusion development office.

Hiroshi Kishimoto, an executive director of JAERI, says the network will contribute to 'satellite research' for validating ITER design, for example the development of a heat exchanger for converting the energy in the high-energy neutrons formed in the fusion process.
But he emphasizes that the planned network for ITER would have to take a more technological approach than the Monbusho-led network, which is focused mainly on academic research.

Despite US withdrawal from the ITER programme, and waning enthusiasm among other partners, Japan - which last year suggested plans for a scaled-down version of the reactor to meet budget constraints - is still keen to host the facility (Nature 394,3; 1998).

But some researchers are concerned at the financial implications of Japan's enthusiasm for hosting ITER. "The reactor is not expected to begin its operation until the middle of the next decade, and we don't even know whether it will work," says one leading nuclear physicist. "We need to think carefully before making a commitment that could not only jeopardize basic research in fusion science but also worsen the nation's financial situation."

Asako Saegus

\section{... as Europeans lobby for reactor construction}

Munich

European proponents of the International Thermonuclear Experimental Reactor (ITER) have launched a campaign to win support for the construction of the fusion reactor in the run-up to key budget decisions due next year.

The first of a series of public seminars took place in Munich last month, introducing the concept of a slimmed-down version of ITER intended to meet criticism of excessive cost. Last week ITER scientists held an exhibition at the European Parliament and lobbied members of the parliament, many of whom have expressed scepticism of fusion as a viable energy source.

The lobbying comes at a time when the ITER collaboration - a partnership between Europe, Japan and Russia - is preparing a detailed design outline for the reactor before the opening in Japan next spring of budgetary discussions for 2001.

The design will also form the basis of a proposal for funding under the European Commission's sixth Framework programme of research (FP6), due to start in 2003. Discussions about the content of FP6 begin next summer.

Plasma physicists in the collaboration believe that the engineering design phase for ITER should not be further extended. It has already been extended for an additional three years to cope with cash shortages resulting from waning political confidence (see Nature 387, 746; 1997). The physicists believe the time has come to bite the bullet and start building, to test the validity of

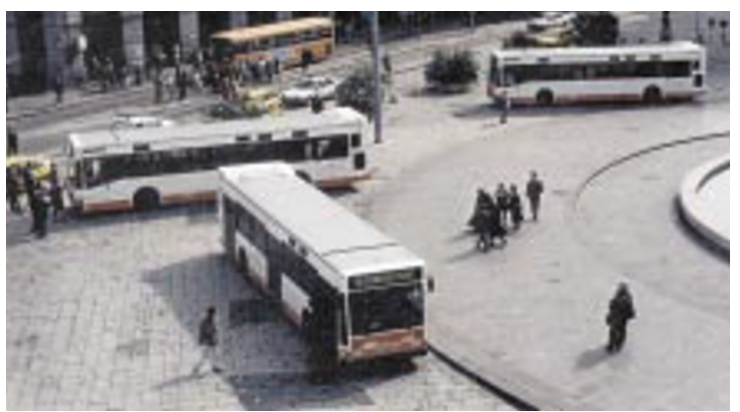

Street demo: technology developed from fusion research is being used in the energy-efficient Altrobus in Bologna, Italy.

experimental plasma-physics predictions.

"Plasma-physics research will lose its dynamism without a close coupling to energy production," Umberto Finzi, director of the European Commission's energy programme, told the Munich meeting.

The ITER programme suffered a major blow earlier this year when the United States withdrew support. The remaining three partners are now promoting a concept, known informally as ITER-Lite, which reduces technical objectives and cost.

The cost of ITER-Lite, around US $\$ 3$ billion, is less than half that of the original proposal, but the original goal of achieving ignition has been dropped. Ignition is an important phenomenon for physicists. But, to move confidently to the next step of a demonstration fusion power plant, ITER has only to demonstrate gain - energy amplification.

Both Japan and Canada (on behalf of the European partner) are hoping to offer sites to host ITER during the next couple of years. Klaus Pinkau, recently retired director of the Max Planck Institute for Plasma Physics in Garching, told the Munich meeting he was optimistic that, once a site had been established, the United States would consider rejoining. Pinkau is co-chairman of a working group set up to oversee the design of a cheaper ITER.

A site in Canada would probably be more attractive to the United States because its geographical proximity would favour participation of US industry in the construction.

But the United States will need considerable persuasion to change its position. It argues that it does not need to look for a new, cheap source of power because it can rely on its own oil supplies.

It also has a substantial commitment to research into inertial confinement fusion, an approach that competes with ITER's magnetic confinement approach. But Pinkau said that the United States should recognize the political importance of ensuring stable energy supplies in other countries. Alison Abbott 